\title{
SIMULATION-BASED MODELLING OF THE IMPACT OF RIDESHARING ON URBAN SYSTEM
}

\author{
Sun, J.*; Liu, S. F.*; Zhang, X. H.** \& Gong, D. Q.* \\ ${ }^{*}$ School of Economics and Management, Beijing Jiaotong University, Beijing 100044, China \\ ${ }^{* *}$ School of Science, Beijing Jiaotong University, Beijing 100044, China \\ E-Mail: 20120568@bjtu.edu.cn
}

\begin{abstract}
This paper follows the basic principles of taxi dynamics and uses an agent-based modelling approach $(\mathrm{ABM})$ to simulate the underlying road network, vehicles and passenger situations in an urban environment and to carry out evaluations. First, based on two evaluation metrics: passenger travel time and total number of passengers transported, a comparison is made between the ride-hailing system (RH) and the system with ridesharing under different supply and demand rates, revealing the impact of ridesharing on system efficiency at urban scale. Then, in the ridesharing system (RS), four levels of supply are taken as examples, and passenger travel time is disaggregated to quantify how the definition of vicinity in the matching process affects the passenger travel time, passenger waiting time and vehicle detour time in a given city. Finally, a summary of the level of supply and the range of vicinity where ridesharing can be effective is presented in terms of the total distance travelled by the system. From a city management perspective, these results can help in the analysis and planning of efficient transport services.

(Received in September 2021, accepted in January 2022. This paper was with the authors 3 weeks for 1 revision.)
\end{abstract}

Key Words: Ride-Hailing, Ridesharing, Agent-Based Model, Simulation

\section{INTRODUCTION}

As an essential infrastructure in the urban transport system, public transport can effectively carry a larger number of passengers and reduce the negative externalities of private car travel. However, many residents do not have access to effective public transport services due to its defined routes and departure times. In this case, they often meet their travel needs by means of transport such as walking and taking taxis. Therefore, it is necessary to promote the development of the taxi industry.

The traditional taxi drivers pick up passengers by waiting at a fixed location or searching aimlessly on the street, while passengers can only access the service through roadside cabs. With the rapid development of travel platforms such as DIDI and Uber in recent years, online taxis have become an emerging industry in the industry. Passengers' travel needs and drivers are dispatched by the platform, big data technology enables the uncertainty to be effectively reduced and the operational efficiency is higher. Driven by the current and future development trends of the information age, online taxis dispatched by platforms should occupy a pivotal position in the travel service system for urban residents.

In recent years, along with the rapid growth of the sharing economy and the increased focus on energy use and emissions in the transport sector, ridesharing services have evolved and dynamic ridesharing systems have emerged. Traditional taxis, if they can offer ridesharing services at all, tend to be driver-led, pulling passengers with similar destinations at a number of hotspots, an approach that is inefficient and does not offer economies of scale. With the rapid development of information technology today, commuters can access ridesharing services with the help of travel platforms such as DIDI and Uber. By aggregating travel demand with similar origins and destinations and matching vehicles and passengers with similar routes, the platforms ensure that ridesharing services are effectively managed and operated on a city-wide basis, promoting sustainable urban transport development. Overall, ridesharing services in ride- 
hailing platforms are emerging in innovative forms due to the emergence of new technologies that offer an alternative between traditional car services and public transport services [1].

Our contributions are: (1) a model incorporating individual passenger characteristics is presented using the ABM approach; (2) the efficiency changes of urban system before and after the inclusion of ridesharing service are compared to understand the potential effectiveness of ridesharing services and its scope of application; (3) a discussion of the phased changes brought about by supply under determined demand, and the effect of matching spatial extent on passenger time costs and system mileage savings rates is investigated at different levels of supply.

\section{LITERATURE REVIEW}

Many early studies focused on carpool studies with the same origin and/or destination [2]. In recent years, a demand-responsive, real-time dynamic ride-sharing service has become increasingly popular due to the development of information technology. Dong et al. recognized the need for online taxis to provide services as a complement to the taxi industry based on data related to the Chinese mobile travel platform DIDI [3]. Wang et al. showed that taxi pooling reduces passenger waiting time and travel time during peak hours, promotes an increase in travel demand, and has a significant reduction in the average distance travelled by vehicles [4].

Much of the existing research in the field of ride-sharing focuses on the optimization of two types of algorithms, vehicle-passenger matching and vehicle travel paths. Gargiulo et al. designed a dynamic ride sharing service that achieves optimal matching in a short period of time by adopting an improved strategy [5]. Lee and Savelsbergh studied the matching problem between passengers and dedicated or temporary drivers by building an integer programming with the objective of minimum total cost [6]. Masoud and Jayakrishnan proposed an efficient solution to the many-to-many matching problem [7]. Gu et al. studied the co-multiplication problem for individual vehicles with the objectives of minimizing the number of vehicles and minimizing the total distance travelled by vehicles [8]. Yu et al. formulated the optimal route problem as a Markov decision process (MDP) problem in pursuit of long-term profits, while solving it using numerical iteration [9]. Li et al. developed a path-based equilibrium model in the form of a variational inequality, and then transformed the variational inequality into an equivalent mixed complementary problem by combining a heuristic routing algorithm to determine shared travelable paths and optimize them to alleviate congestion [10]. The ridesharing problem studied by some scholars considered both matching and optimal route selection, but their optimization goal is different from the previous ones, which is seeking to maximize the average load rate of the whole system [11]. Zhang et al. modelled the assignment problem as an online weighted matching problem and designed a heuristic nearest neighbour algorithm to solve the driver's travel path [12]. Jindal et al. focused on developing an effective carpooling strategy with the goal of maximizing traffic efficiency and reducing traffic congestion [13].

While ridesharing is undoubtedly positive by any measure, there is a lack of empirical evidence on the impact of ridesharing on consumers in different areas and at different levels of accessibility. Lam et al. found that consumer surplus from ridesharing varied significantly by area [14]. Based on this, the paper discusses its impact on system service levels with different vicinity ranges in Section 4. In addition to the two types of optimization algorithm studies discussed above, many scholars have also explored ride-sharing systems using simulation approaches, of which agent-based approaches are the most widely used.

The ABM approach allows for a more realistic and natural transition to the overall performance evaluation of the system by describing the behaviour of microscopic subjects and the interactions between them [15]. Nourinejad and Roorda showed the positive role of dynamic 
ride-sharing in terms of user cost and vehicle kilometre travelled (VKT) savings by designing centralized (binary integer programming) and decentralized (dynamic auction-based multiagent) optimization algorithms to match passengers and drivers [16]. Lokhandwala and Cai used agent-based modelling to carry out simulations comparing traditional taxi ride-sharing (with frequency) and shared autonomous taxi models [17]. Markov et al. designed a simulation of an on-demand mobility service based on the City of Chicago, Illinois, to find a socially optimal service design [18]. Thaithatkul et al. investigated the existence of day-to-day homogeneity by simulating the dynamics of a smart ridesharing system (SRS) [19]. Inturri et al. constructed an agent-based simulation model, compared Demand Responsive Shared Transport (DRST) with taxi services and applied it to a real case in Italy [20]. Wang studied the subway emergency evacuation based on a simulation model of Agent and Pathfinder [21].

Related scholars have also given interactions between online hailing platforms and other transport modes. Qian and Ukkusuri studied the competition between online hailing platforms and traditional taxis [22]. Berrada and Poulhès discussed the interaction between DRT (ondemand responsive ridesharing service) and CPT (conventional public transit) and their impact on the economy [23]. In addition, with the rapid development of autonomous vehicle in recent years, people began to focus on the related research of AVS and proved its effectiveness. Zhao et al. used numerical experiments to demonstrate that autonomous vehicles can encourage commute carpooling and significantly reduce costs [24]. Fagnant and Kockelman studied that dynamic ride-sharing in a fully automated system effectively reduced users' average service times and travel costs and affected their travel times and routes to become more flexible [25].

\section{URBAN SYSTEM SIMULATION}

For ease of discussion, the simple simulated ride-hailing system without ridesharing is denoted as RH (ride-hailing system) and the simulated system with ridesharing added is denoted as RS (ridesharing system).

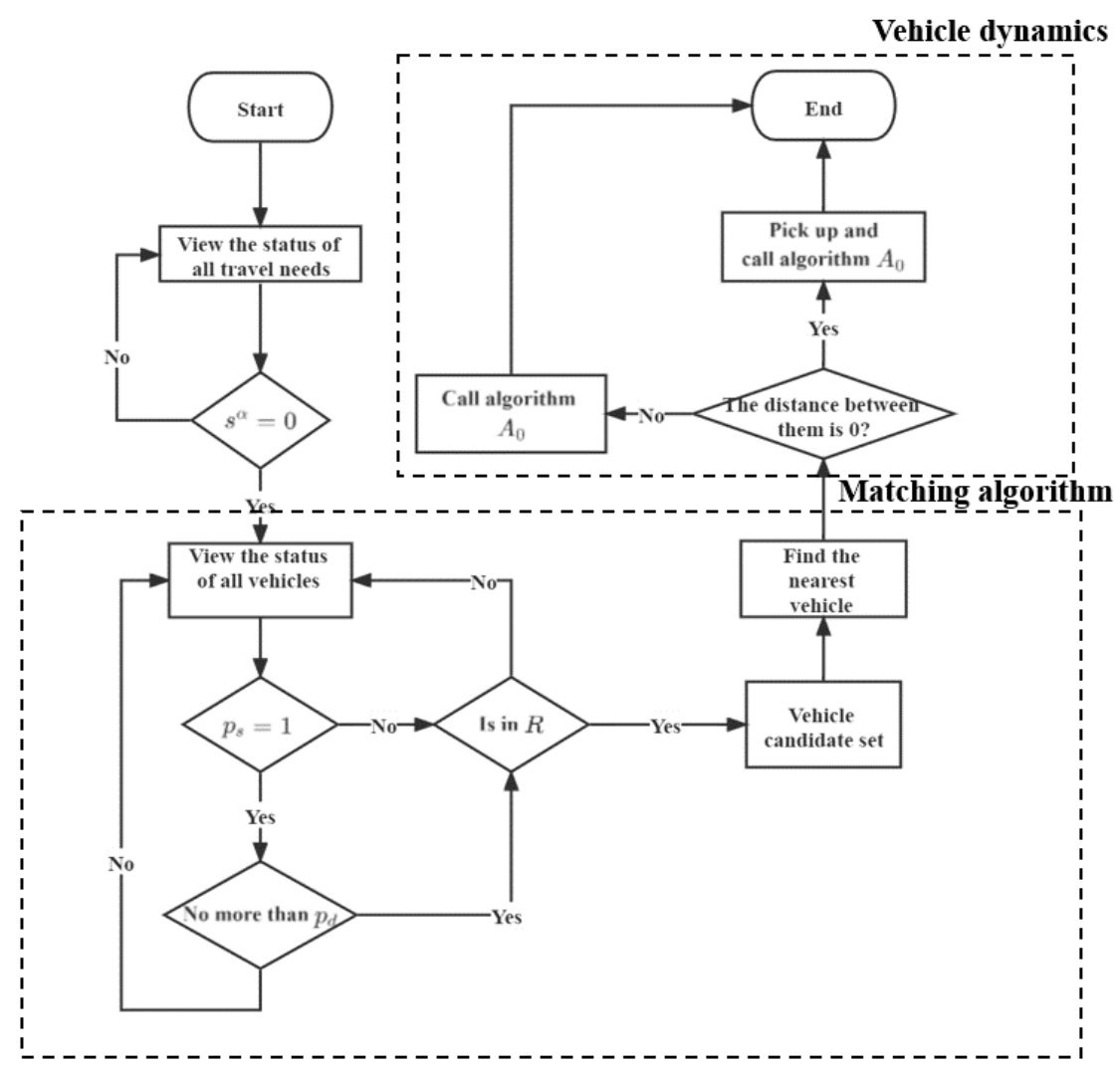

Figure 1: Matching process and the vehicles dynamic process. 


\subsection{Agent-based modelling (ABM)}

The components in the model are shown below.

Table I: The notations, meanings and comments of each component involved in the simulation.

\begin{tabular}{|c|c|c|}
\hline Notations & Meanings & Comments \\
\hline $\mathrm{G}\left(N_{1}, N_{2}, N_{3}\right)$ & Urban road network & $\begin{array}{l}\text { Static network, dividing the size of city and blocks. } N_{1} \text { is the } \\
\text { number of all nodes in the x-direction of the city boundary, and } \\
N_{2} \text { is the number of all nodes in the y-direction of the city } \\
\text { boundary, and } N_{3} \text { is the number of points in the block. The inner } \\
\text { part of the block is the infeasible area and, graphically, the } \\
\text { urban network is shown in Fig. } 2 \text {. }\end{array}$ \\
\hline$R$ & Vicinity range & I \\
\hline$N_{m}$ & Total simulation time & I \\
\hline$N_{v}$ & Vehicle number & Constant in each complete simulation. \\
\hline$p^{\alpha}$ & Passenger travel demand & $\begin{array}{l}\text { Each passenger corresponds to a travel demand at the time of } \\
\text { generation and contains a random origin and destination in the } \\
\text { form of coordinates in the } \mathrm{x} \text { and y directions. }\end{array}$ \\
\hline$p_{s}$ & Ride sharing willingness & $\begin{array}{l}p_{s}=0 \text { means no preference for ridesharing, } p_{s}=1 \text { represents } \\
\text { agreement to ridesharing. All demands in } \mathrm{RH} p_{s}=0 \text {, each travel } \\
\text { demand in RS is generated corresponding to a random } p_{s} .\end{array}$ \\
\hline$p_{d}$ & Detourtolerance of passengers & $\begin{array}{l}\text { In the RS system, each shared travel demand is generated } \\
\text { corresponding to a random } p_{d}, 0 \leq p_{d} \leq 1 \text {, in units of } \pi \text {. }\end{array}$ \\
\hline$\left(R_{p}, t_{p}\right)$ & $\begin{array}{l}\text { (Demand generation rate, } \\
\text { Demand generation interval) }\end{array}$ & $R_{p}$ new passenger demand generated every $t_{p}$ time. \\
\hline
\end{tabular}

Passenger status: $s^{\alpha}=0$ means no match has been generated, $s^{\alpha}=1$ means that a match has been generated, $s^{\alpha}=2$ for boarded, and $s^{\alpha}=3$ means the service is complete.

Before the simulation starts, the number of vehicles, seat capacity and speed are set and remain constant during the $N_{m}$ period. The vehicle seat capacity is 2 . In this paper, all vehicles are empty before passengers are generated in the system at the initial moment, roaming randomly through the city at a speed of 1 . In addition, we introduce $s^{i}(t)$ to characterize the states of the vehicle. $s^{i}(t)=0$ means that the vehicle is empty and $s^{i}(t)=1$ means the vehicle has been matched and is on its way to pick up a passenger, $s^{i}(t)=2$ means the vehicle is available for sharing, $s^{i}(t)=3$ means that the vehicle is not available for sharing. Where $s^{i}(t)=3$ includes two cases: there is no more capacity or there is a passenger on board who does not want to share.

For vehicle dynamics, we use $A_{0}$ and $A_{e}$ to represent the travel routing algorithms for occupied or booked vehicles and empty vehicles, respectively. Algorithm $A_{0}$ refers to the fact that the vehicle always runs to the target location by the shortest path principle from the current location, which is applicable to the two situations of vehicle pickup and drop-off. Algorithm $A_{e}$ describes the random roaming situation of empty vehicles, that is, their speed direction at the next moment is always random until it is matched again.

\subsection{Matching algorithm}

At each moment the system will find a suitable vehicle for a passenger demand with $s^{\alpha}=0$. If the status, direction of travel and location of a vehicle meet certain conditions, then it can be entered into the candidate set for the current demand, and the vehicle closest to the passenger in the candidate set will be matched directly. Fig. 2 can briefly describe the processes of determining whether the travel direction and the location of the vehicle are qualified, in this paper, a circular area is used to represent the vicinity range $R$. 


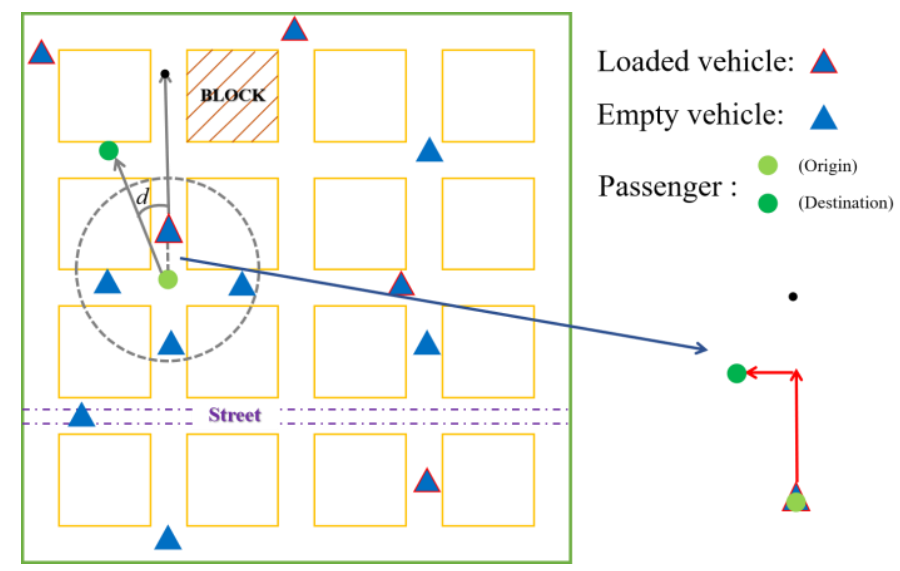

Figure 2: In the urban network, using a ridesharing demand as an example, describes the supply and demand matching determination process, and the pick-up and drop-off process of vehicles.

Assuming that at this point in time the shared demand $p^{\alpha}$ is in the matching process. Since the three empty vehicles do not have a fixed route, but are in a random state of travel, and they are located within $R$, they are candidates for this demand. For the other vehicle already carrying passengers, if $s^{i}(t)=2$, we define two vectors $\overrightarrow{t_{1}}$ and $\overrightarrow{t_{2}}$ to make a determination of its travel direction, $\overrightarrow{t_{1}}$ is directed from the departure point of demand $p^{\alpha}$ to its destination and $\overrightarrow{t_{2}}$ is directed from the current position of the vehicle to its destination, i.e. the destination of the passengers already on board. When the angle $d$ formed by the two vectors is less than the $p_{d}$ corresponding to the demand $p^{\alpha}$, the vehicle is considered to be traveling in the same direction as destination direction of $p^{\alpha}$ and can be a member of the matching candidate set when it is within range $R$. For this example, since the loaded vehicle is closest to $p^{\alpha}$, the two are successfully matched. Note that, the vehicle will then change its original path of travel to pick up the newly matched second passenger first, and then compare which one to drop off first. After receiving $p^{\alpha}$ and following the algorithm $A_{0}$ to the fork in the road, it is easy to see that the vehicle is closer to the second passenger's destination and therefore delivers the second passenger first, the travel path following algorithm $A_{0}$ is shown as the red line in the small diagram on the right in Fig. 2. What can be seen is that outside the range $R$, there are many other vehicles on the city streets either roaming empty or in working condition, driving independently of each other.

\subsection{Performance indicators}

The following five indicators are used to compare the RH and RS, highlighting the impact of ridesharing:

(1) Total passenger travel time $T_{p}$,

(2) Total number of passengers transported $N_{p}$,

(3) Passenger waiting time $W_{p}$,

(4) Detour time $D_{v}$,

(5) Mileage saving rate $S_{v}(\%)$.

$T_{p}$ is the sum of the time taken by all passengers receiving the service from generation to completion of the service. $N_{p}$ refers to the total number of passengers transported by vehicles during the $N_{m}$ period. $W_{p}$ means the total waiting time from generation to boarding for all passengers during the $N_{m}$ period. $D_{v}$ is counted for shared rides, if the loaded vehicle is successfully matched with a second passenger and the distance from its departure point is not zero, a detour is required to pick up the second passenger, which requires a detour time to be counted for the passenger on board. $S_{v}$ calculates a ratio to represent the percentage of mileage savings from sharing in the system compared to the original non-ridesharing. 


\section{NUMERICAL EXPERIMENTS}

All experiments in this section are based on the same parameters: a regular square grid of $200 \times 200$ and $5 \times 5$ for cities and blocks, respectively, i.e. $\mathrm{G}(200,200,5)$, and $N_{p}=50000$. In addition, for intuitive analysis, the horizontal and vertical coordinates of most of the following figures of simulated results are in logarithmic form.

\subsection{Total passenger travel time $T_{p}$}

This section discusses the effects of supply changes on $T_{p}$ for two demand levels: $\left(R_{p}, t_{p}\right)=$ $(50,100)$ and $(20,100)$, where $R=2$. The results are shown in Fig. 3.

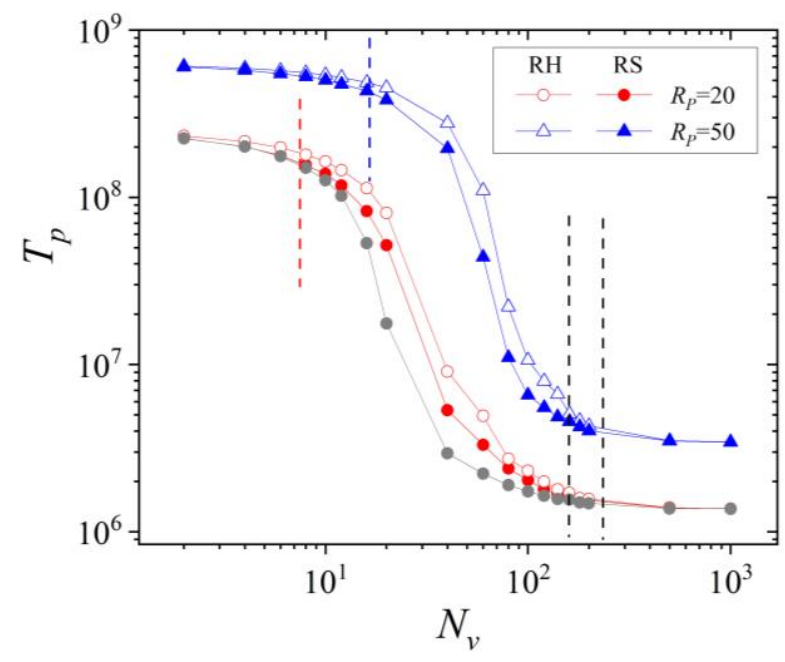

Figure 3: In the RH and RS systems, the variation of $T_{p}$ with $N_{v}$ at two demand rates.

$T_{p}$ tends to increase with $N_{v}$ in a flat-sharply decreasing-flat trend of change, the $T_{p}$ of $R_{p}=50$ is always higher than the $R_{p}=20$ case, and overall the RS system corresponds to a lower $T_{p}$ than the RH system. When supply increases in a small range in the early part of the period, demand is greater than supply, when demand is not effectively met and the vast majority of passengers remain waiting, and due to the long waiting times, the $T_{p}$ continues to be at a high level and there is no obvious downward trend, we write down the end of this phase as $N_{v}^{1}$. As the supply increases further, $T_{p}$ goes through three stages of slow decline, rapid decline, and once it reaches the point where the second order derivative equals zero, slow decline again. The change in the phase is heavily dependent on the number of vehicles. When the supply continues to increase to saturation, the supply is greater than the demand and when new passengers are generated in the system, there are always vehicles available and the demand for travel can all be met effectively, at which point increasing the number of vehicles has little effect on reducing waiting times and $T_{p}$ is no longer dependent on the number of vehicles, nor does it change significantly with the number of vehicles, but is only determined by the shortest distance between the origin and destination of each OD pair. At this stage, the reduction in passenger waiting times is mainly due to an increase in the density of available vehicles, a reduction in the average distance between available vehicles and passengers, and the fact that more vehicles are to some extent able to provide fewer detours, reducing the trip time of shared passengers. The match ability of demand at a limited urban scale increases, but the marginal reduction in waiting times and trip times is very small. We denote the phase of the supply turnaround from a downward trend to a flat trend as $N_{v}^{*}$ and several factors, such as demand rate and whether or not to share, can have an impact on $N_{v}^{*}$. When $N_{v}^{1}<N_{v}<N_{v}^{*}$, an increase in supply can lead to a significant reduction in passenger waiting times, while when $N_{v}>N_{v}^{*}$, the marginal benefit to passengers is small. 
In the RS system, the overall level of $T_{p}$ is lower than the RH system, and the curve is able to not only start the decline phase faster, but can also reach the supply saturation phase much earlier. When passengers choose to accept shared travel, the range of vehicles that can be matched is somewhat expanded, as loaded vehicles can then also be matched with them, rather than just empty ones, and passengers are able to get on faster, reducing their waiting time. On the other hand, the same number of vehicles can meet more demand when shared, so supply can have an impact on passenger waiting times earlier, reaching the saturation stage in smaller supply numbers. It is important to note that at low levels of supply in the early stages, even when ridesharing exists, the impact on $T_{p}$ is not significant, whereas after reaching the saturation stage, even without ridesharing, the number of vehicles far exceeds the current demand, so that there is no significant difference in the curves corresponding to $\mathrm{RH}$ and $\mathrm{RS}$ systems at these two stages.

In addition, for the two defined demand rate scenarios of $R_{p}=20$ and $R_{p}=50$, at $R_{p}=50$, RS performs better than RH in terms of the maximum reduction achieved on $T_{p}$, ridesharing brings more obvious advantages under high demand. The grey line in Fig. 3 simulates the situation when all passengers agree to ridesharing at $R_{p}=20$, when the overall level of $T_{p}$ improves substantially compared to the RH system, this is a good indication of the positive effects of ridesharing.

\subsection{Total number of passengers transported $N_{p}$}

In this section, the impact of supply changes on $N_{p}$ is studied at the determined demand level of $\left(R_{p}, t_{p}\right)=(50,100)$, where $R=2$. The simulation results are shown below.

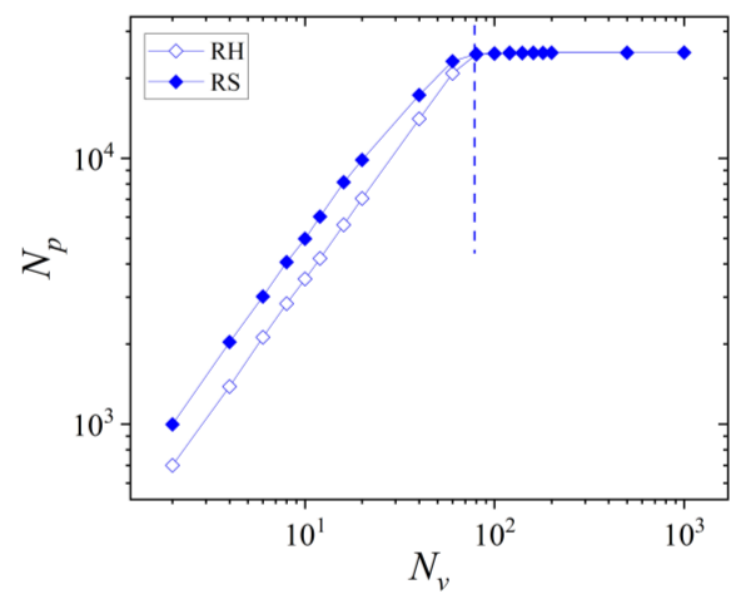

Figure 4: In both RH and RS systems, the impact of $N_{p}$ when $N_{v}$ changes.

When the number of vehicles is at a low level, around $N_{v}<80, N_{p}$ always increases with $N_{v}$ increases, and the $N_{p}$ curve of RS system is always higher than that of the RH system. This is because the number of vehicles is at the stage $N_{v}^{1}<N_{v}<N_{v}^{*}$ in Fig. 3, undersupply, a small increase in the number of vehicles is able to effectively satisfy the demand in the system that is waiting, and the total number of passengers that the system can serve increases, $N_{p}$ will rise more significantly. In addition, when passengers accept a shared ride, one vehicle can pull two passengers at the same time, the same number of vehicles have a higher service capacity and therefore the $N_{p}$ of RS system is always higher. The performance of $N_{p}$ depends on the $N_{v}$ in the system and the presence or absence of ridesharing.

When the supply continues to increase to 80 , the total number of passengers that can be served by the system no longer changes significantly, $N_{p}$ is maintained at a high level and the two curves overlap. Because the system has reached steady state at this point, i.e. the sum of demand $N_{p}^{\prime}$ in the three states of $s^{\alpha}=0, s^{\alpha}=1$ and $s^{\alpha}=2$ fluctuates around a steady value, as 
shown in Fig. 5, the number of passengers in the RH system is significantly higher than in the RS system. Even if the service level could reach a higher stage, passenger demand for vehicles is limited and $N_{p}$ cannot continue to rise any further. At this point, although the two systems diverge when the supply is insufficient in the first, after $N_{v}>80$, the performance of $N_{p}$ depends on $R_{p}$.

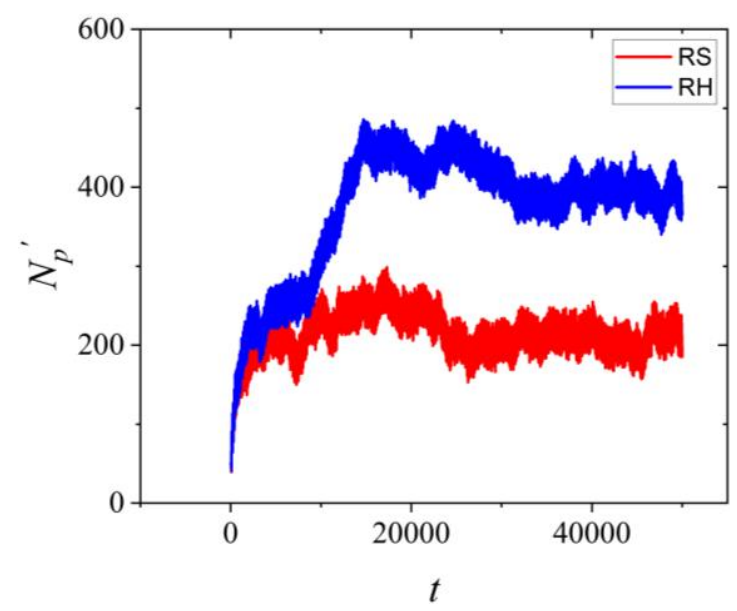

Figure 5: When $N_{v}=80$, during the $N_{m}$ period, the variation of $N_{p}^{\prime}$ with time.

\subsection{Effect of $R$ on efficiency in RS systems}

In this section, in the demand scenario of $\left(R_{p}, t_{p}\right)=(50,100)$, changes of relevant metrics when $R$ changes are discussed. From the analysis in Section 4.1, it can be seen that there is a transition from shortage to saturation in supply for a defined demand, therefore, in this section we first select four supply levels of 50,100, 200 and 500, discussing the effect of $R$ on $T_{p}$, as shown in Fig. 6. Then the decomposition of $T_{p}$ is carried out, from these two perspectives of passenger waiting time $W_{p}$ and detour time $D_{v}$, further discussion is made, as shown in Fig. 7.

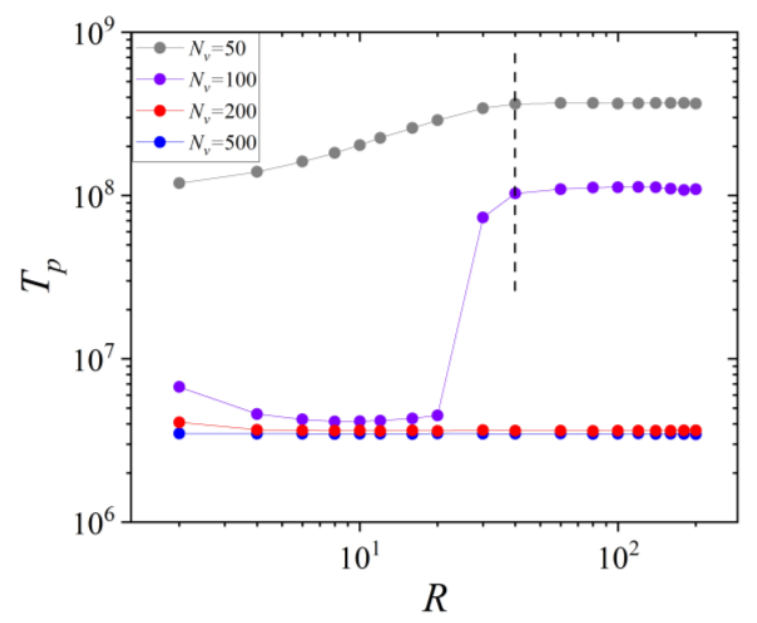

Figure 6: Under four levels of supply, the change of $T_{p}$ with $R$.

When $N_{v}=50$, with the increase of $R$, in the first period, $T_{p}$ is on an upward trend and later reaches stability. In addition, the overall level of $T_{p}$ is also the highest among the four supply levels. In such a situation of extreme shortage of supply, even if there is a shared service, system demand cannot be effectively met, the passenger waiting time in the system itself is a high level, and the expansion of the matching range brought about by the expansion of the vicinity range at this time will instead cause the vehicles in the system in a shared state to be occupied for a long time in order to meet multiple demands, and the detour time increases, and the 
continuous waiting of passengers not only prevents the demand from being met and even drives $W_{p}$ to increase slightly within its own high level, as shown in Fig. 7 a. When $R$ keeps expanding, it can be interpreted as a limiting situation in which any vehicle within the city boundary that satisfies the remaining conditions can be matched with a passenger, at which point even if a vehicle is located in the farthest corner of the city from the passenger, the driver will rush to pick him up and the passenger will have to wait passively, $T_{p}, W_{p}$ and $D_{v}$ all remain at a consistently high level.
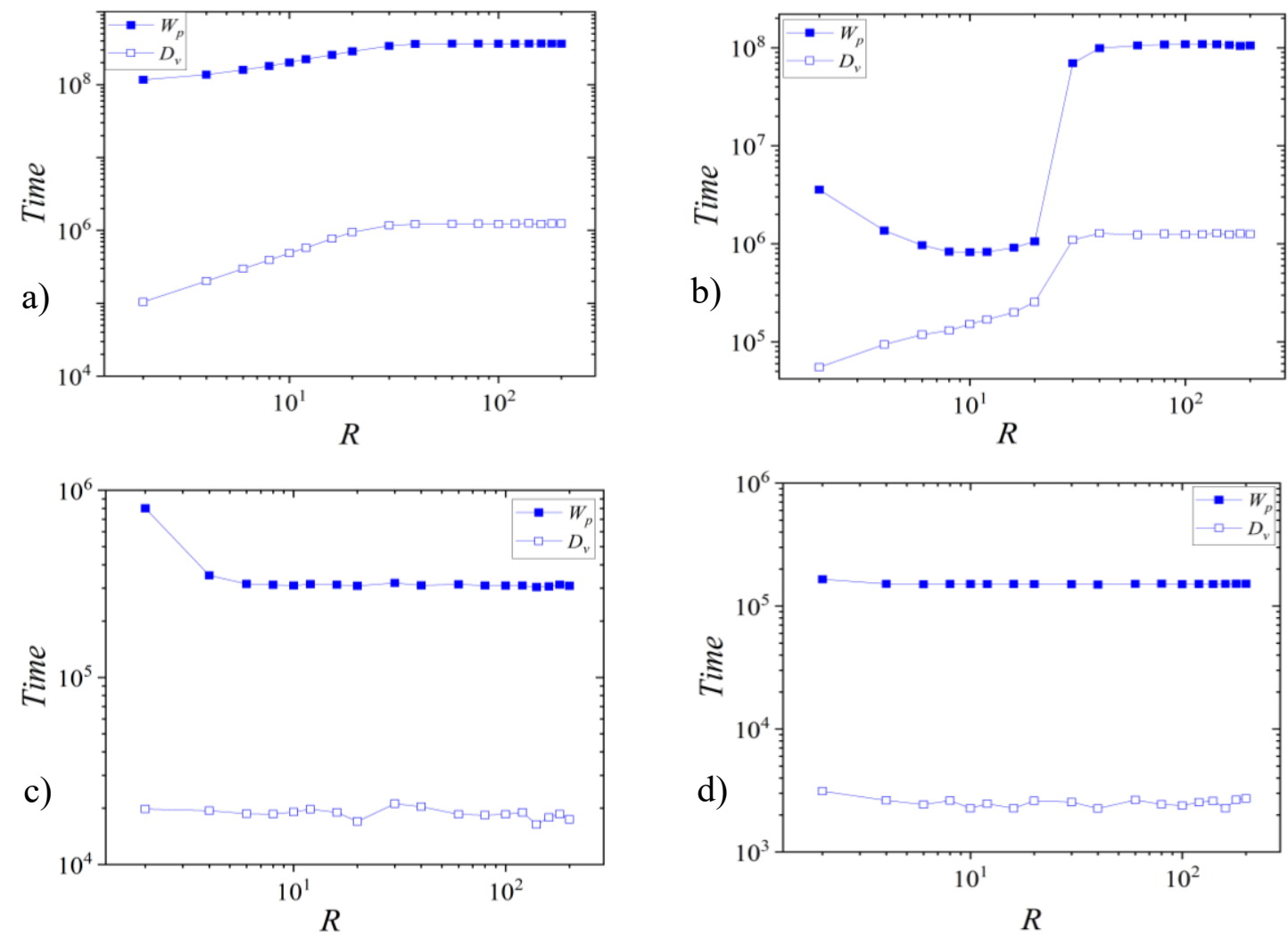

Figure 7: The change of $W_{p}$ and $D_{v}$ with $R$; a) $N_{v}=50$, b) $N_{v}=100$, c) $N_{v}=200$, d) $N_{v}=500$.

When the level of supply increases further, in the case of $N_{v}=100, T_{p}$ shows a trend of first decreasing, then increasing and finally reaching stability, similar to the case of $N_{v}=50, T_{p}$ basically stabilizes around $R=40$. In addition, as the current supply level is also significantly increased relative to the level of $N_{v}=50$, the overall level of $T_{p}$ is also significantly decreased. As can be seen from Fig. 7 b, both sharing and matching range expansions can lead to further reductions in $W_{p}$ within certain limits. When $R$ increases in the early stage, although the average distance between matchable passengers and vehicles increases and $D_{v}$ rises, the number of matching vehicles in the vicinity of passengers rises, and the resource usage caused by detours does not have a relative impact on the service demand process in the system, and may even play a positive role, as these vehicles are detoured to pick up new passengers. This means that on an individual level, the passengers on board may have to endure a detour, but on an overall level it is beneficial to the system, However, when $20<R<30$, the change of $D_{v}$ and $W_{p}$ is consistent with a rapid upward trend, which is consistent with the matching occupancy at $N_{v}=50$ mentioned above. When $R$ continues to grow, the extreme case described above is reached, and to a certain extent, $D_{v}$ is also consistent with the high level of $W_{p}$.

For the current level of demand, when the optimal supply for the transition phase is reached, i.e. in the $N_{v}=200$ scenario, it can be seen from Fig. 6 that at this point the level of $T_{p}$ is no different from $N_{v}=500$, is the lowest of the four supply scenarios, and does not change significantly with increasing $R$. When $N_{v}=200$, only when $R$ increases in a small range (less 
than or equal to 6), there is a downward trend of $W_{p}$ and $D_{v}$ does not change significantly in this phase, sufficient supply weakens the influence of $R$ and ridesharing. When $N_{v}=500$, the supply is saturated and the average distance between vehicles and passengers within the city is very small. At this time, $W_{p}$ and $D_{v}$ are significantly lower than in the other three supply levels and no longer fluctuate significantly with increasing $R$.

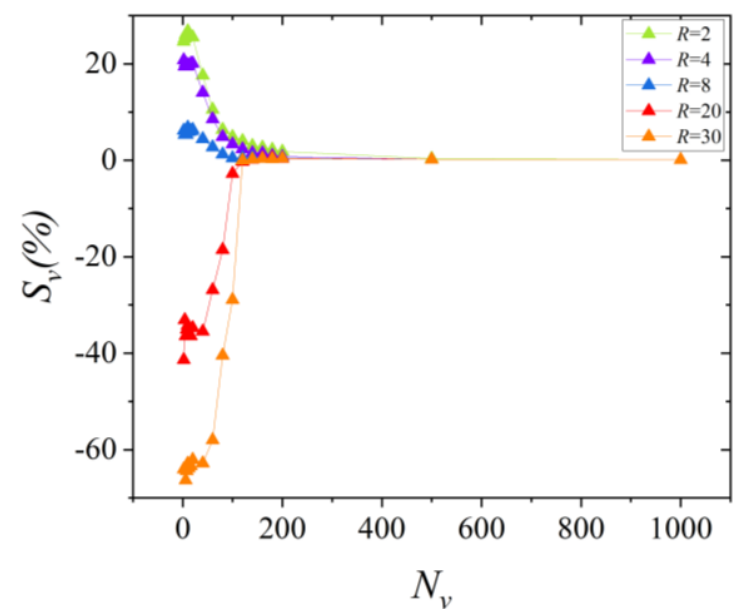

Figure 8: At five different levels of $R$, the change of $S_{v}$ with $N_{v}$.

From the perspective of the distance travelled and fuel consumption of the system, the variation of mileage saving rate $S_{v}$ with the number of vehicles $N_{v}$ is explored, as shown in Fig. 8. As can be seen from the discussion above, no matter what the supply level is, when $R$ increases to about $30, T_{p}$ does not change significantly. Therefore, we mainly select five cases, $R=2,4,8,20$ and 30, for discussion.

As can be seen from the discussion above, the situation when there is a shortage of supply and the vicinity range is large is more problematic because if a loaded vehicle matches to a passenger who is far away, it takes the driver a long time to take a detour to pick him up and this move is actually inefficient, this can be seen from Fig. 8, when $R=20$ and $R=30$ are at a low level of $N_{v}$. When the supply is low, once the vehicle is occupied by the detour bind, not only do passengers continue to wait, but sharing is instead less efficient compared to the original non-ridesharing scenario. The total distance travelled is not only not reduced, but the increase in detour distance makes $S_{v}$ as low as around $-60 \%$, and the situation is worse for $R=30$ than $R=20$ due to the wider matching range. This negative effect diminishes as the shortage is alleviated with increased supply. Conversely, in the three small vicinity ranges of $R=2,4,8$, successful matches are closer to passengers and vehicles, so detour distances are usually not too far away, and sharing can play a positive role in reducing the total distance travelled when supply is low in the first period; moreover, the lower the $R$, the higher the level of $S_{v}$. As supply increases further until saturation is reached, the mileage saving effect of sharing becomes more limited, and eventually the five curves coincide and reach the same stable level.

\section{CONCLUSIONS AND FUTURE RESEARCH DIRECTIONS}

In this paper, we model the vehicle and passenger agents in an urban system based on ABM in the context of online ride-hailing, design vehicle routing algorithms and matching algorithms, test the changes in the service level of the system before and after adding ridesharing for different combinations of supply and demand, and carry out corresponding numerical simulations. Two main indicators are used to compare the two transport systems: total passenger travel time and the total number of passengers transported. The first metric can be used to measure system service levels and passenger experience perceptions, while the second can be 
seen as a measure of system efficiency. As different passengers have different preferences for different time components, we further disaggregate the passenger travel time and discuss the impact of vicinity range on passenger travel time, passenger waiting time and detour time in a system that incorporates ridesharing. In addition, the variation of the total distance travelled is also discussed from a sustainability perspective.

The results discussed above show that testing different service configurations in a simulated environment is necessary to provide a basis for urban transport planning and ridesharing service design. In future studies, we plan to test more combinations of service configurations in the hope of reaching more generalized conclusions. Discussing the role of the main reasons affecting passengers' ridesharing willingness (e.g. waiting times, pricing policies, etc.) in the performance of urban transport systems [26]. In addition, by including travel mode options such as public transport, walking, etc. in the system, user preferences can be encapsulated in the model and more realistic situations can be discussed.

\section{ACKNOWLEDGEMENTS}

This work was supported by Beijing Social Science Foundation under Grant 19JDGLA002, 18JDGLA018, 21JCB093, 21JCC092, MOE (Ministry of Education in China) Project of Humanities and Social Sciences under Grant 19YJC630043, the National Natural Science Foundation of China under Grant J1824031, the National Social Science Fund of China under Grant 21FGLB059 and was partially supported by Beijing Logistics Informatics Research Base. We appreciate their support very much. The work presented in this study remains the sole responsibility of the authors.

\section{$\underline{\text { REFERENCES }}$}

[1] Inturri, G.; le Pira, M.; Giuffrida, N.; Ignaccolo, M.; Pluchino, A.; Rapisarda, A.; D'Angelo, R. (2019). Multi-agent simulation for planning and designing new shared mobility services, Research in Transportation Economics, Vol. 73, 34-44, doi:10.1016/j.retrec.2018.11.009

[2] Barth, M.; Todd, M. (1999). Simulation model performance analysis of a multiple station shared vehicle system, Transportation Research Part C: Emerging Technologies, Vol. 7, No. 4, 237-259, doi:10.1016/s0968-090x(99)00021-2

[3] Dong, Y.; Wang, S.; Li, L.; Zhang, Z. (2018). An empirical study on travel patterns of internet based ride-sharing, Transportation Research Part C: Emerging Technologies, Vol. 86, 1-22, doi:10.1016/j.trc.2017.10.022

[4] Wang, Y.; Zheng, B.; Lim, E.-P. (2018). Understanding the effects of taxi ride-sharing - a case study of Singapore, Computers, Environment and Urban Systems, Vol. 69, 124-132, doi:10.1016/ j.compenvurbsys.2018.01.006

[5] Gargiulo, E.; Giannantonio, R.; Guercio, E.; Borean, C.; Zenezini, G. (2015). Dynamic ride sharing service: are users ready to adopt it?, Procedia Manufacturing, Vol. 3, 777-784, doi:10.1016/ j.promfg.2015.07.329

[6] Lee, A.; Savelsbergh, M. (2015). Dynamic ridesharing: Is there a role for dedicated drivers?, Transportation Research Part B: Methodological, Vol. 81, 483-497, doi:10.1016/j.trb.2015.02.013

[7] Masoud, N.; Jayakrishnan, R. (2017). A decomposition algorithm to solve the multi-hop Peer-toPeer ride-matching problem, Transportation Research Part B: Methodological, Vol. 99, 1-29, doi:10.1016/j.trb.2017.01.004

[8] Gu, Q.-P.; Liang, J. L.; Zhang, G. (2021). Approximate ridesharing of personal vehicles problem, Theoretical Computer Science, Vol. 871, 30-50, doi:10.1016/j.tcs.2021.04.009

[9] Yu, X.; Gao, S.; Hu, X.; Park, H. (2019). A Markov decision process approach to vacant taxi routing with e-hailing, Transportation Research Part B: Methodological, Vol. 121, 114-134, doi:10.1016/j.trb.2018.12.013

[10] Li, Y.; Liu, Y.; Xie, J. (2020). A path-based equilibrium model for ridesharing matching, Transportation Research Part B: Methodological, Vol. 138, 373-405, doi:10.1016/ j.trb.2020.05.007 
[11] Hou, L.; Li, D.; Zhang, D. (2018). Ride-matching and routing optimisation: Models and a large neighbourhood search heuristic, Transportation Research Part E: Logistics and Transportation Review, Vol. 118, 143-162, doi:10.1016/j.tre.2018.07.003

[12] Zhang, C.; Xie, J.; Wu, F.; Gao, X.; Chen, G. (2020). Pricing and allocation algorithm designs in dynamic ridesharing system, Theoretical Computer Science, Vol. 803, 94-104, doi:10.1016/ j.tcs.2019.05.045

[13] Jindal, I.; Qin, Z. T.; Chen, X.; Nokleby, M.; Ye, J. (2018). Optimizing taxi carpool policies via reinforcement learning and spatio-temporal mining, 2018 IEEE International Conference on Big Data, 1417-1426, doi:10.1109/bigdata.2018.8622481

[14] Lam, C.; Liu, M.; Hui, X. (2021). The geography of ridesharing: a case study on New York City, Information Economics and Policy, Vol. 57, Paper 100941, 18 pages, doi:10.1016/ j.infoecopol.2021.100941

[15] Calabrò, G.; Inturri, G.; le Pira, M.; Pluchino, A.; Ignaccolo, M. (2020). Bridging the gap between weak-demand areas and public transport using an ant-colony simulation-based optimization, Transportation Research Procedia, Vol. 45, 234-241, doi:10.1016/j.trpro.2020.03.012

[16] Nourinejad, M.; Roorda, M. J. (2016). Agent based model for dynamic ridesharing, Transportation Research Part C: Emerging Technologies, Vol. 64, 117-132, doi:10.1016/j.trc.2015.07.016

[17] Lokhandwala, M.; Cai, H. (2018). Dynamic ride sharing using traditional taxis and shared autonomous taxis: a case study of NYC, Transportation Research Part C: Emerging Technologies, Vol. 97, 45-60, doi:10.1016/j.trc.2018.10.007

[18] Markov, I.; Guglielmetti, R.; Laumanns, M.; Fernández-Antolín, A.; de Souza, R. (2021). Simulation-based design and analysis of on-demand mobility services, Transportation Research Part A: Policy and Practice, Vol. 149, 170-205, doi:10.1016/j.tra.2021.01.004

[19] Thaithatkul, P.; Seo, T.; Kusakabe, T.; Asakura, Y. (2017). Simulation approach for investigating dynamics of passenger matching problem in smart ridesharing system, Transportation Research Procedia, Vol. 21, 29-41, doi:10.1016/j.trpro.2017.03.075

[20] Inturri, G.; Giuffrida, N.; Ignaccolo, M.; le Pira, M.; Pluchino, A.; Rapisarda, A.; D'Angelo, R. (2021). Taxi vs. demand responsive shared transport systems: an agent-based simulation approach, Transport Policy, Vol. 103, 116-126, doi:10.1016/j.tranpol.2021.01.002

[21] Wang, F. (2021). Multi-scenario simulation of subway emergency evacuation based on multi-agent, International Journal of Simulation Modelling, Vol. 20, No. 2, 387-397, doi:10.2507/IJSIMM20$\underline{2-\mathrm{CO} 8}$

[22] Qian, X.; Ukkusuri, S. V. (2017). Taxi market equilibrium with third-party hailing service, Transportation Research Part B: Methodological, Vol. 100, 43-63, doi:10.1016/j.trb.2017.01.012

[23] Berrada, J.; Poulhès, A. (2021). Economic and socioeconomic assessment of replacing conventional public transit with demand responsive transit services in low-to-medium density areas, Transportation Research Part A: Policy and Practice, Vol. 150, 317-334, doi:10.1016/ j.tra.2021.06.008

[24] Zhao, Y.; Guo, X.; Liu, H. (2021). The impact of autonomous vehicles on commute ridesharing with uncertain work end time, Transportation Research Part B: Methodological, Vol. 143, 221248, doi:10.1016/j.trb.2020.11.002

[25] Fagnant, D. J.; Kockelman, K. M. (2018). Dynamic ride-sharing and fleet sizing for a system of shared autonomous vehicles in Austin, Texas, Transportation, Vol. 45, No. 1, 143-158, doi: $10.1007 / \mathrm{s} 11116-016-9729-\mathrm{z}$

[26] Suryani, E.; Hendrawan, R. A.; Adipraja, P. F. E.; Indraswari, R. (2020). System dynamics simulation model for urban transportation planning: a case study, International Journal of Simulation Modelling, Vol. 19, No. 1, 5-16, doi:10.2507/IJSIMM19-1-493 